\title{
Axonal integrity predicts cortical reorganisation following cervical injury
}

\author{
Patrick Freund, ${ }^{1,2,3,4}$ Claudia A Wheeler-Kingshott, ${ }^{5}$ Zoltan Nagy, ${ }^{2}$ Nikos Gorgoraptis, ${ }^{1}$ \\ Nikolaus Weiskopf, ${ }^{2}$ Karl Friston, ${ }^{2}$ Alan J Thompson, ${ }^{1}$ Chloe Hutton ${ }^{2}$
}

'Department of Brain Repair \& Rehabilitation, UCL Institute of Neurology, UCL, London, UK ${ }^{2}$ Wellcome Trust Centre for Neuroimaging, UCL Institute of Neurology, UCL, London, UK ${ }^{3}$ Spinal Cord Injury Centre, Royal National Orthopaedic Hospital, UCL, London, UK

${ }^{4}$ Swiss Paraplegic Research, Nottwil, Switzerland

${ }^{5}$ Department of

Neuroinflammation, UCL Institute of Neurology, UCL, London, UK

Correspondence to Dr Patrick Freund, Department of Brain Repair and Rehabilitation, UCL Institute of Neurology, Queen Square, London WC1N 3BG, UK;

p.freund@ucl.ac.uk

Received 22 November 2011 Accepted 6 March 2012 Published Online First 6 April 2012

\section{ABSTRACT}

Background Traumatic spinal cord injury (SCI) leads to disruption of axonal architecture and macroscopic tissue loss with impaired information flow between the brain and spinal cord-the presumed basis of ensuing clinical impairment.

Objective The authors used a clinically viable, multimodal MRI protocol to quantify the axonal integrity of the cranial corticospinal tract (CST) and to establish how microstructural white matter changes in the CST are related to cross-sectional spinal cord area and cortical reorganisation of the sensorimotor system in subjects with traumatic SCl.

Methods Nine volunteers with cervical injuries resulting in bilateral motor impairment and 14 control subjects were studied. The authors used diffusion tensor imaging to assess white matter integrity in the CST, T1-weighted imaging to measure cross-sectional spinal cord area and functional MRI to compare motor task-related brain activations. The relationships among microstructural, macrostructural and functional measures were assessed using regression analyses.

Results Diffusion tensor imaging revealed significant differences in the CST of SCl subjects-compared with controls - in the pyramids, the internal capsule, the cerebral peduncle and the hand area. The microstructural white matter changes observed in the left pyramid predicted increased task-related responses in the left M1 leg area, while changes in the cerebral peduncle were predicted by reduced cord area.

Conclusion The observed microstructural changes suggest trauma-related axonal degeneration and demyelination, which are related to cortical motor reorganisation and macrostructure. The extent of these changes may reflect the plasticity of motor pathways associated with cortical reorganisation. This clinically viable multimodal imaging approach is therefore appropriate for monitoring degeneration of central pathways and the evaluation of treatments targeting axonal repair in $\mathrm{SCl}$.

\section{INTRODUCTION}

Following traumatic spinal cord injury (SCI) axonal architecture is disturbed and information flow between the brain and spinal cord is compromised, often resulting in severe clinical impairment. ${ }^{1}$ The spinal $\operatorname{cord}^{2-4}$ and cranial grey matter $(G M)^{4-6}$ become atrophic. However, changes in the white matter (WM) containing the corticospinal tract
(CST), whose integrity is critical for manual dexterity, ${ }^{7}$ are less well understood. In particular, three key questions remain unanswered: (1) Can CST integrity and demyelination be measured comprehensively? (2) How do microstructural changes in CST relate to macroscopic changes (ie, cord area)? (3) Is a reduction in CST integrity associated with changes in cortical motor function?

Microstructural changes can be quantified using diffusion-tensor imaging (DTI); a technique that provides in vivo information about tissue microstructure. ${ }^{8}$ Specifically, fractional anisotropy (FA) has been reported to be a marker of both axonal count $^{9}$ and myelin content. ${ }^{10}$ Axial diffusivity (AD) and radial diffusivity $(\mathrm{RD})$, which report the ease of water diffusion along or across the axons, are thought to reflect the integrity of axons and myelin, respectively. ${ }^{11}$ Importantly, $\mathrm{AD}$ measured immediately after onset of spinal cord injury has been shown to correlate with spared axons and to predict locomotor recovery in a mouse model of SCI. ${ }^{12}$ Thus, $\mathrm{AD}$ and $\mathrm{RD}$ have the potential to report the axonal integrity more comprehensively than FA and the directionally averaged diffusion coefficient.

In the brain of paraplegic subjects, abnormalities within areas containing the CST have been demonstrated using standard DTI metrics. ${ }^{6}{ }^{13}$ Furthermore, evidence for cortical reorganisation following SCI has been provided by functional MRI (fMRI). ${ }^{14}$ However, the relationships between microstructural white matter changes measured comprehensively using DTI, spinal changes and brain activations have not been investigated.

In a previous study, ${ }^{4}$ we have shown that the degree of cortical reorganisation in SCI subjects-measured by fMRI-is predicted by spinal atrophy; that is lower cross-sectional spinal cord area (SCA) and is related to clinical impairment. In the present study, we used DTI data from the same cohort to investigate microstructural changes in the CST using voxel-based morphometry of cranial DTI data (FA, $\mathrm{AD}, \mathrm{RD}$ and mean diffusivity $(\mathrm{MD})$ ). Using regression analyses, we examined the relationship between microstructural changes, and the previously observed SCA reduction and functional reorganisation in the motor cortex. ${ }^{4}$ We show marked differences in FA, $\mathrm{AD}$ and $\mathrm{RD}$ between SCI subjects and controls and demonstrate clear relationships between these microstructural metrics and measures of macrostructure and functional reorganisation in the motor cortex and both spinal and cortical levels. 


\section{METHODS}

\section{Subjects}

We studied the same ten right handed subjects with SCI (one subject was excluded because of incomplete MRI data) reported previously. ${ }^{4}$ Their mean age was 47.5 years, (SD 11.3 years, range $=29-61$ years, $n=9$ ) and they met the following inclusion criteria: (1) Upper and lower limb impairment; (2) No head or brain lesion associated with the trauma leading to the injury; (3) No history of seizure, medical or mental illness; (4) no MRI contraindications. We also studied fourteen right-handed healthy subjects (mean age $=40.14$ years, $\mathrm{SD}=16.18$, range $=25-71$ ). The mean ages of the control and SCI groups were not significantly different ( $t$-test: $p=0.24$ ). All participants gave informed, written consent before the study, which was approved by the Joint Ethics Committee of the UCL-Institute of Neurology and the National Hospital for Neurology and Neurosurgery (ref: 08/0243).

All participants were scanned using MRI, and clinically assessed on the 9-Hole Peg Test (9HPT), maximum voluntary contraction (MVC) and Arm Action Research Test (ARAT). The average of two trials for each hand of the 9HPT and of the MVC of the dominant hand was calculated. Three SCI subjects were unable to complete the 9HPT within the maximum time allowed (300 s) with their non-dominant hand. Differences in motor performance between SCI subjects and controls were assessed with a two-sample t-test (implemented in 17.0 (SPSS Inc.)). A (corrected) $\mathrm{p}$ value $<0.05$ was considered significant.

\section{Image acquisition and analysis}

Subjects were scanned on a 1.5T whole-body scanner (Sonata, Siemens Medical Systems, Erlangen, Germany) operated with a radio frequency body transmit and an 8-channel receive-only head coil. Structural imaging, fMRI and DTI were all performed on the same day. Image processing and voxel-wise statistical analyses were performed using SPM8 (http://www.fil.ion.ucl.ac. $\mathrm{uk} / \mathrm{spm} /$ ), unless otherwise stated.

\section{Structural image acquisition}

The structural image acquisition was previously described, ${ }^{4} 16$ but is briefly detailed here for completeness. A $3 \mathrm{D}$ whole-brain structural image volume, including brain stem and high cervical cord was acquired using an optimised T1-weighted (T1w) modified driven equilibrium Fourier transform (MDEFT) sequence. $^{16} 17$ The imaging parameters were: isotropic $1 \mathrm{~mm}^{3}$ resolution, field of view (FoV) $=256 \times 256 \mathrm{~mm}^{2}$, acquisition matrix $=256 \times 256,176$ sagittal partitions, repetition time $(\mathrm{TR})=12.24 \mathrm{~ms}$, echo time $(\mathrm{TE})=3.56 \mathrm{~ms}$, inversion time $(\mathrm{TI})=$ $530 \mathrm{~ms}$, flip angle $=23^{\circ}$, fat saturation, bandwidth $=106 \mathrm{~Hz} /$ Pixel and an acquisition time of $13 \mathrm{~min}$ and $43 \mathrm{~s}$. All images were visually checked for artefacts before further processing.

\section{Measurement of cross-sectional spinal cord area}

Cross-sectional spinal cord area measurement has been described previously. ${ }^{4}$ This involved identifying the $\mathrm{C} 2$ disc-as the caudal reference-on the individual $\mathrm{T} 1 \mathrm{w}$ volumes. A region of interest (ROI) was drawn around the cord cerebrospinal fluid (CSF) space and cord on five axial-oblique slices $(3 \mathrm{~mm}$ slice thickness), reformatted perpendicular to the main cord axis. The SCA was measured automatically, using a semi-automated segmentation method. ${ }^{18}$ A two-sample t-test was used to test for a difference in SCA between SCI subjects and controls (SPSS V.17.0). $\mathrm{p}$ values $<0.05$ were considered significant.

Functional imaging of the brain

The fMRI acquisition, paradigm and processing were previously described in detail. ${ }^{4}$ Functional MRI was performed using a custom-made echo-planar imaging (EPI) sequence. ${ }^{19}$ The imaging parameters were: in-plane resolution $=3 \times 3 \mathrm{~mm}^{2}$, FoV $=192 \times 192 \mathrm{~mm}^{2}$, acquisition matrix $=64 \times 64,48$ axial slices acquired sequentially in descending order, slice thickness $=2 \mathrm{~mm}$, interslice gap $=1 \mathrm{~mm}, \mathrm{TE}=50 \mathrm{~ms}, \mathrm{TR}=4.3 \mathrm{~s}$, flip angle $=90^{\circ}$, readout bandwidth $\mathrm{BW}=2298 \mathrm{~Hz} /$ Pixel.

\section{Functional MRI paradigm}

The fMRI paradigm consisted of six repetitions of active pseudorandomised $20 \mathrm{~s}$ blocks of right sided (1) repetitive isometric handgrip, (2) electrical median nerve stimulation at the medial wrist and (3) electrical tibial nerve stimulation at the medial malleolus alternating with $20 \mathrm{~s}$ rest blocks. ${ }^{4}$ Participants lay supine and viewed visual stimuli projected via a mirror system onto a frosted screen. Participants were cued to exert $30 \%$ of their maximum voluntary contraction level. Visual feedback about the amount of force and the duration of handgrip was provided on the screen in the form of a thermometer $(1.7 \mathrm{~s})$. In total, 48 handgrips were performed, using an MRI compatible grip manipulandum. ${ }^{20}$ Prior to scanning, participants practiced the motor task until comfortable. Immediately prior to scanning, the target force of $30 \%$ of maximum voluntary contraction was derived for each individual from two trials (measured while supine in the scanner). Since the aim of the current study was to investigate the impact of degenerative axonal changes on taskrelated activation within the motor cortex, we only used the handgrip task related responses for the subsequent analyses. Results for the other conditions are presented in our previous article. $^{4}$

\section{Functional MRI processing and analysis}

Functional images were reconstructed off-line, realigned and unwarped $^{21}$ to account for movement- and susceptibilityinduced image distortions, slice-time corrected, normalised to the Montreal Neurological Institute (MNI) anatomical standard space, and smoothed spatially using an isotropic Gaussian kernel with $8 \mathrm{~mm}$ full width at half-maximum (FWHM). Motion was $<2.5 \mathrm{~mm}$ in all subjects.

A general linear model (GLM) was constructed that consisted of box-car stimulus functions encoding the three (blocked) conditions convolved with a haemodynamic response function. ${ }^{22}$ Temporal derivatives were also included to accommodate latency and slice timing effects. The GLM was used to calculate a contrast of parameter estimates at each voxel of the fMRI time series from each subject reflecting task-related activation. These were used as summary statistics for subsequent between-subject (random effects) analysis of hand-grip activations, to produce SPMs of the t-statistic.

\section{DTI acquisition}

DTI was performed using a single shot echo planar imaging sequence employing the twice refocused spin-echo method for diffusion encoding. ${ }^{23}$ Two data sets were collected with alternating phase encoding blip directions to allow for the correction of susceptibility induced geometric distortions. ${ }^{24}$ Each data set consisted of 61 images with a b-value $=1000 \mathrm{~s} / \mathrm{mm}^{-2}$ and 7 images with a $b$-value $=100 \mathrm{~s} / \mathrm{mm}^{-2}$. The diffusion encoding gradient directions were distributed evenly on the surface of the unit sphere. ${ }^{25}$ The imaging parameters were: $2.3 \mathrm{~mm}^{3}$ isotropic resolution, FoV $=220 \times 220 \mathrm{~mm}^{2}$, matrix $=96 \times 96,60$ axial slices, no inter-slice gaps, interleaved slice acquisition order, slice-toslice repetition time $=160 \mathrm{~ms}, \mathrm{TE}=90 \mathrm{~ms}$, flip angle $=90^{\circ}$, readout bandwidth $=2003 \mathrm{~Hz} /$ Pixel and a total acquisition time of approximately $19 \mathrm{~min}$. The two data sets were corrected for 
movement artefacts and combined into a single dataset to minimise susceptibility induced geometric distortions. ${ }^{24}$ This data set was used to fit a diffusion tensor at each voxel using the RESTORE method ${ }^{26}$ as implemented in Camino (http://www. camino.org). ${ }^{27}$ Maps of $\mathrm{FA}, \mathrm{AD}, \mathrm{RD}$ and $\mathrm{MD}$ (henceforth $\mathrm{DTI}$ maps), were estimated for each subject.

Pre-processing for voxel-based morphometry of DTI data

$\mathrm{T} 1 \mathrm{w}$ images were bias corrected and segmented into GM, WM and CSF tissue probability maps. After co-registering each FA map to the corresponding WM map, the parameters were used to transform all DTI maps to the space of the corresponding T1w images. A WM mask was created and multiplied by each registered DTI map yielding WM specific maps of DTI metrics. The GM and WM tissue probability maps were used to nonlinearly transform each subject's masked DTI maps into standard MNI space. ${ }^{28}$ A tissue specific weighted Gaussian smoothing (FWHM $10 \mathrm{~mm}$ ) was applied to the masked DTI maps, preserving the value of the DTI metrics. ${ }^{29}$

\section{Regions of interest}

Our primary aim was to characterise degenerative changes of the CST that may be caused by trauma to the spinal cord. To this end, we defined an ROI representing the CST from the Johns Hopkins University (JHU) white-matter tractography atlas (figure 1). ${ }^{30}$ We then defined the following bilateral regions containing the CST from the International Consortium for Brain Mapping (ICBM)-DTI-81 WM labels atlas ${ }^{31}$ : pyramids, cerebral peduncle and posterior limb of the internal capsule (figure 1). To define ROIs in the motor cortex, we centred $10 \mathrm{~mm}^{3}$ spheres on coordinates of peak activation from an fMRI study of limb function. ${ }^{32}$ Coordinates were, for the left $M 1$ hand $(x=-38, y=-26, z=52)$ and leg area $(x=-16, y=-40, z=64)$ and right $M 1$ hand $(x=38, y=-24, z=50)$ and leg area $(x=12$, $y=-38, z=64)$.

Statistical analysis of DTI data

A GLM was constructed that consisted of the group (SCI) effect, with age and total intracranial volume (TIV) as nuisance covariates. The TIV was calculated from the sum of the GM, WM and CSF probability maps and was included to account for any confounding (non-specific) effects of overall brain size.

The GLM was fitted to every voxel in the brain for each DTI metric (FA, MD, $\mathrm{AD}$ and $\mathrm{RD}$ ) and t-statistics were calculated for the parameters estimated from the GLM, indicating evidence for differences between SCI and control subjects in the presence of nuisance variables. The t-tests were one-tailed and the associated $p$ values were corrected for multiple comparisons of all voxels within the whole brain and then within each ROI (described above), (Family Wise Error (FWE) $p<0.05$ ), using Gaussian random field theory. ${ }^{22}$ Performing the FWE correction over the whole brain provided a descriptive overview of effects Performing the FWE correction over each a priori defined ROI provided a more sensitive-hypothesis driven-search for significant effects. Only significant results $(p<0.05$, corrected) are reported.

To explore how changes in DTI metrics are related to SCA and disease duration in the SCI subjects, we first identified CST
Figure 1 The corticospinal tract region was derived from the JHU whitematter tractography atlas and is shown as a red overlay on orthogonal slices through the brain. The bottom right figure indicates the coronal locations of the bilateral regions containing the pyramids (violet), cerebral peduncle (cyan) and the internal capsule (yellow) from the ICBM-DTI-81 white-matter labels atlas. The blue and green circles indicate the approximate locations of the functional M1 leg and hand areas respectively.

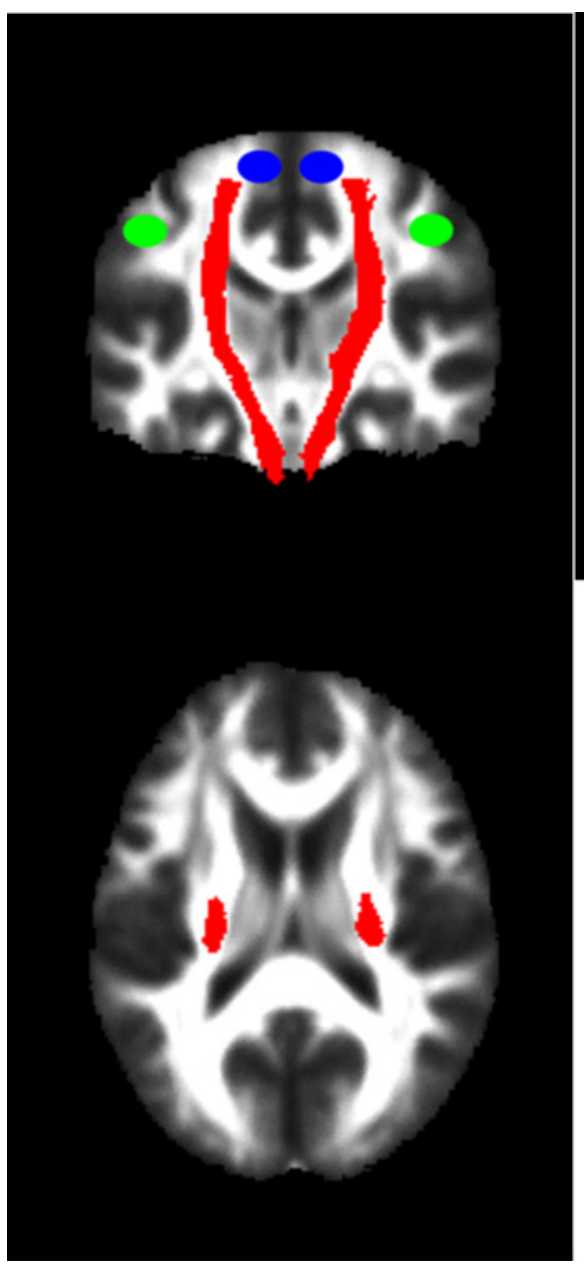


Table 1 Clinical and behavioural data for the subjects with $\mathrm{SCl}$ and controls

\begin{tabular}{|c|c|c|c|c|c|c|c|c|}
\hline & Age & $\begin{array}{l}\text { Time since } \\
\text { injury (years) }\end{array}$ & $\begin{array}{l}\text { ASIA-upper limb } \\
\text { motor score }\end{array}$ & $\begin{array}{l}\text { ASIA-lower limb } \\
\text { motor score }\end{array}$ & ARAT & dh 9HPT (sec.) & ndh 9HPT (sec.) & MVC (mV) \\
\hline \multicolumn{9}{|c|}{$\mathrm{SCl}(\mathrm{n}=9)$} \\
\hline Mean & 47.1 & 14.6 & 26.93 & 19.5 & 38.72 & 98.77 & 141.72 & 0.16 \\
\hline SD & 10.7 & 6.91 & 10.87 & 16 & 13.93 & 84.45 & 121.44 & 0.15 \\
\hline \multicolumn{9}{|c|}{ Controls $(n=14)$} \\
\hline Mean & 40.1 & & & & & 16.8 & 18.1 & 0.53 \\
\hline SD & 16.2 & & & & & 1.8 & 2.0 & 0.23 \\
\hline
\end{tabular}

ARAT, Arm research arm test; ASIA, American Spinal Injury Association; dh, dominant hand; ndh, non-dominant hand; MVC, maximum voluntary contraction; SCl, spinal cord injury; 9HPT, Nine Hole Peg Test.

regions where a significant difference in DTI metric was observed between SCI and control subjects (ie, clusters of contiguous voxels at $\mathrm{p}<0.05$ (FWE corrected)). From these regions, the mean DTI metric (ie, FA, $\mathrm{MD}, \mathrm{AD}$ or $\mathrm{RD}$ ) was extracted. Multiple linear regression analyses (using SPSS) correcting for age, was used to identify independent associations between each of the DTI metrics and SCA and time post injury.

Finally, we tested whether altered DTI metrics predicted cortical reorganisation by investigating how well the DTI metrics explained the activations due to handgrip compared with rest. A GLM was constructed that comprised the group effect, a mean-corrected parametric regressor of interest (ie, the
DTI metric) and age as a nuisance covariate. Parametric regressors were constructed by extracting from all subjects the mean DTI metric (ie, FA, MD, $\mathrm{AD}$ or $\mathrm{RD}$ ) from the CST regions, where a significant difference in DTI metric was observed between SCI and control subjects. A between-subject GLM was used to calculate a $t$-statistic for each voxel using the subject specific motor task activations (contrasts) as dependent variables. This model allowed us to test for the main effect of group, ${ }^{4}$ the main effect of each DTI metric and their interaction. The interaction assessed whether changes in DTI metrics were more sensitive to changes in task-related blood oxygenation level-dependent activation following trauma relative to normal variability.
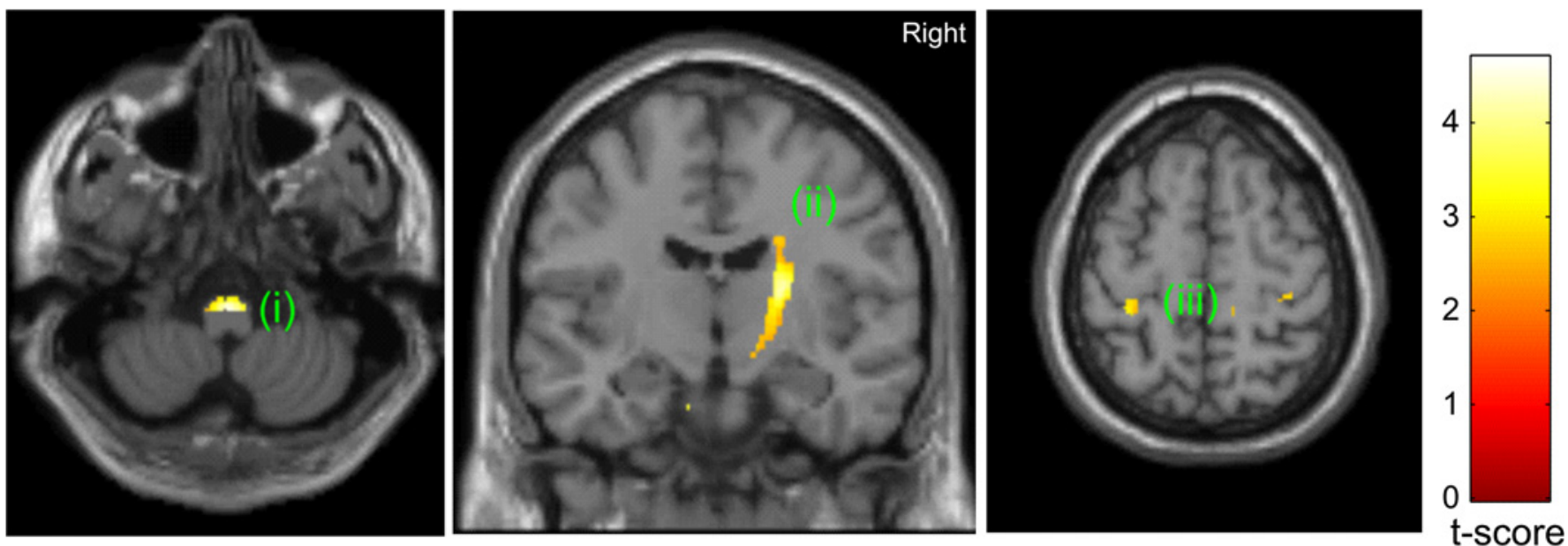

A

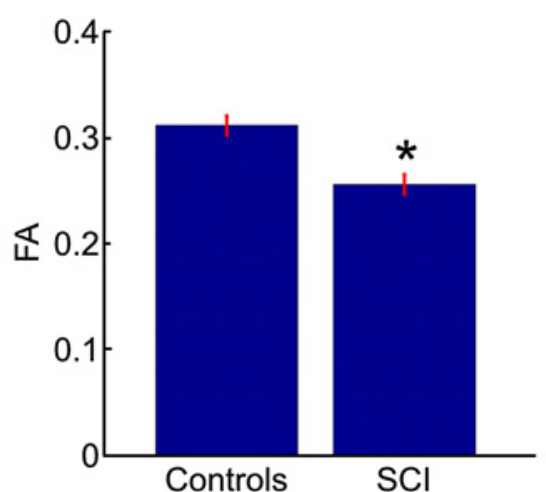

B

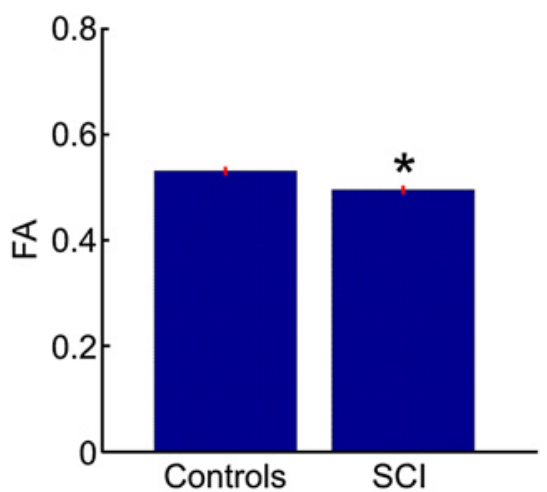

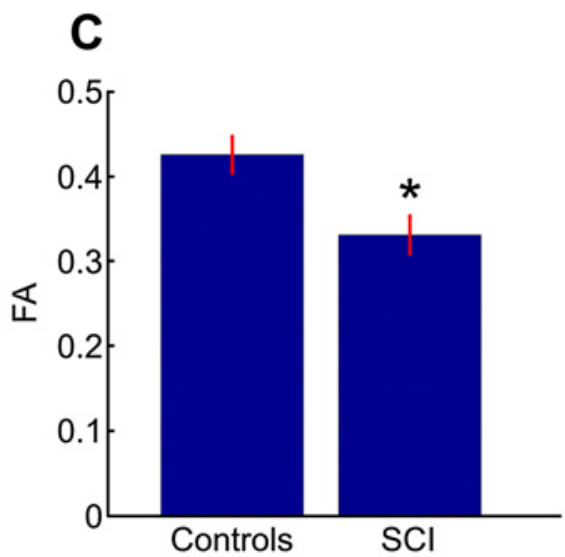

Figure 2 Statistical parametric maps (thresholded at $p<0.01$, uncorrected for display purposes) showing regions of reduced fractional anisotropy (FA) values for spinal cord injury (SCI) subjects compared with controls in the pyramids, the right posterior internal capsule, and left hand area of primary motor cortex. The regions showing reduced FA values are projected onto coronal (middle) and transverse slices (left and right) of the T1w SPM template. The colour bar represents the t-value. Inset A, B, C show the mean FA values of the peak voxel within the cluster of both groups. 


\section{RESULTS}

Clinical data and reduced cross-sectional spinal cord area

Nine male SCI subjects had cervical lesions between C5 and C8 and experienced significant bilateral motor impairment (reduced ASIA (American Spinal Injury Association) motor scores of upper and lower limb and impairment on the ARAT (mean of 38.7 points $(\max =57)$ ), dominant hand ( $\mathrm{t}$-score $=3.3$, degrees of freedom $(\mathrm{df})=21, \mathrm{p}=0.009$, corrected) and non-dominant hand 9HPT ( $\mathrm{t}$-score $=3.6, \mathrm{df}=21, \mathrm{p}=0.006$, corrected) and grip strength (t-score $=4.3, \mathrm{df}=21, \mathrm{p}<0.001$, corrected) compared with controls (table 1). The SCA in SCI subjects was reduced by $33 \%\left(53.1 \mathrm{~mm}^{2}\right.$ (SD 7.68) vs $79.89 \mathrm{~mm}^{2}$ (SD 7.3), t-score=8.3, $\mathrm{df}=21, \mathrm{p}<0.001$ ) compared with control subjects (table 1) and was correlated with measures of manual dexterity as previously reported. $^{4}$

\section{Difference in DTI metrics between SCI subjects and controls}

The whole brain analysis revealed a significant reduction in FA in the right posterior limb of the internal capsule $(p=0.004$, corrected) and left hand area of primary motor cortex ( $p=0.003$; corrected) in SCI compared with control subjects. No significant differences between SCI and control subjects were observed in $\mathrm{MD}, \mathrm{AD}$ and $\mathrm{RD}$ when correcting for multiple comparisons over the whole brain. For the hypothesis driven ROI analyses, decreased FA in the right posterior limb of the internal capsule $(p=0.004$, corrected) and left hand area $(p=0.039$; corrected) were also observed. Furthermore, we found an additional significant reduction in FA in the bilateral pyramids ( $\mathrm{p} \leq 0.008$; corrected) and right leg area of $\mathrm{M} 1$ ( $\mathrm{p}=0.004$; corrected) (figure 2 ) in SCI compared with control subjects. We also found reduced $\mathrm{AD}$ in the right cerebral peduncle $(\mathrm{p}=0.045$; corrected) and increased $R D$ in the right pyramid ( $p=0.048$; corrected) and right cerebral peduncle $(p=0.023$, corrected) (figure 3 ).

Association between DTI metrics, cross-sectional spinal cord area and time post injury

A significant independent relationship was observed between increased $\mathrm{RD}$ in right cerebral peduncle and decreased SCA $\left(r^{2}=0.52, p=0.029\right)$ in SCI subjects (figure 4). No other DTI metric correlated significantly with SCA (table 2). No significant correlation was found with respect to time post injury.

Association between DTI metrics and cortical reorganisation We first confirmed an increase in BOLD activation in the M1 leg area $(p=0.038$; corrected) in SCI subjects compared with controls (figure $5 \mathrm{~A}$ ) as reported previously. ${ }^{4}$ The interaction between the main effect of group and FA was also significant $(p=0.043$; corrected) in the left M1 leg area (figure $5 \mathrm{~B}$ ) suggesting that the dependency between these functional and anatomical measures is stronger post-trauma than predicted by any normal (eg, neurodevelopmental) association. Note the overlap between the regions showing a significant effect of group (figure $5 \mathrm{~A}$ ) and a significant interaction between group and FA (figure 5B) despite the fact that these two effects on task-related activation are orthogonal.
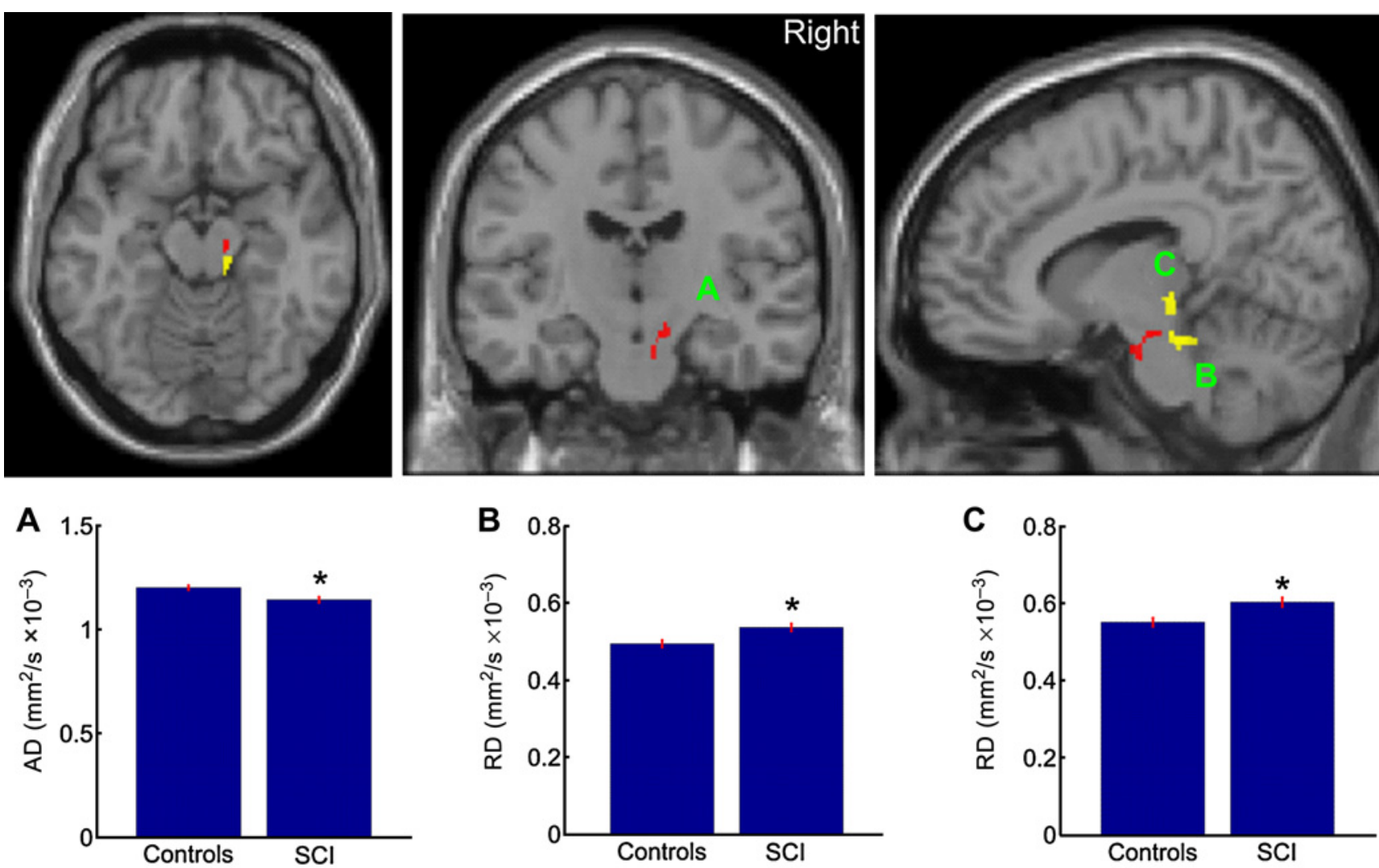

Figure 3 Statistical parametric maps (thresholded at $p<0.01$, uncorrected for display purposes) showing regions of reduced axial diffusivity (AD) (red) and increased radial diffusivity (RD) (yellow) in $(A, B)$ the right pyramid and increased RD in $(C)$ the right cerebral peduncle for spinal cord injury (SCI) subjects compared to controls. The regions showing reduced $A D$ and increased $R D$ are projected onto transverse (left), coronal (middle) and sagittal (right) slices of the T1w SPM8 template. Inset A shows the mean AD values and inset B,C shows the RD values of the peak voxel within the cluster of both groups. 


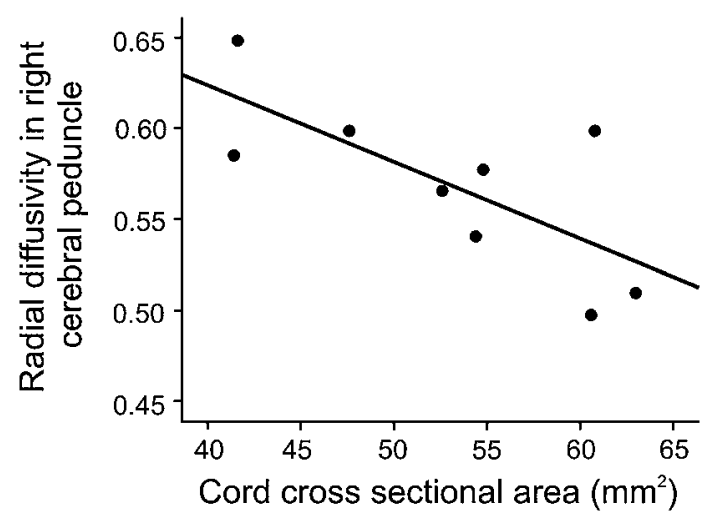

Figure 4 Graph showing the significant correlation between lower cord area and radial diffusivity in cerebral peduncle in spinal cord injury subjects. Note that higher radial diffusivity values correspond to a greater degree of trauma related changes.

\section{DISCUSSION}

This study has shown the potential of $\mathrm{AD}$ and $\mathrm{RD}$ (in addition to FA) to detect axonal disintegration and demyelination in vivo following SCI in man. Crucially, two key findings demonstrated the constructs validity of these measures: (1) a link between peripheral macrostructural (ie, SCA) and central microstructural (ie, DTI indices) measures and (2) a link between central cortical reorganisation (ie, fMRI) and microstructural changes (ie, DTI indices) following traumatic cervical injury. Reduced FA, reduced $\mathrm{AD}$ and increased $\mathrm{RD}$ were observed in regions within the CST, following SCI. Importantly, these results illustrate how DTI metrics measured within the brain could be used as non-invasive biomarkers in clinical trials that target the repair of the injured spinal cord. ${ }^{33}$

\section{FA, AD and RD changes in SCI subjects compared to controls} In agreement with a previous study investigating cranial DTI indices, ${ }^{6}$ FA was reduced within the pyramids, posterior limb of the internal capsule and leg area of the motor cortex. We extended these findings showing reduced FA in the proximity of the left M1 hand-knob area. Animal studies have demonstrated the potential of directional diffusivities (eg, $\mathrm{AD}$ and $\mathrm{RD}$ ) to provide information about the pathological processes, over and above that derived from FA and MD. ${ }^{11}{ }^{34}$ Moreover, the sensitivity of these measures to pathological changes in the spinal cord at sites of focal lesions (and in distal locations) has been demonstrated. ${ }^{35}$ The reduced $\mathrm{AD}$ in the right cerebral peduncle and increased $\mathrm{RD}$ in the right pyramid and cerebral peduncle extended beyond the regions exhibiting reduced FA. The brains of SCI subjects are not affected by focal lesions, therefore changes of directional diffusivities such as $\mathrm{AD}$ and $\mathrm{RD}$ are unlikely to result from biases in the eigenvectors due to localised pathology changes. ${ }^{36}$ The most likely interpretation for lower
$\mathrm{AD}$ and increased $\mathrm{RD}$ in the CST is axonal degeneration ${ }^{37}$ and demyelination. ${ }^{38}$ Decreased $\mathrm{AD}$ has been reported to reflect specifically axonal damage in WM of the spinal cord and brain. ${ }^{39}$ $\mathrm{RD}$, which reflects the degree of demyelination ${ }^{40}{ }^{41}$ was also different between SCI subjects and controls. MD, which reflects the overall extent of structural changes in a voxel and which may be less sensitive, did not differ between SCI subjects and controls. This suggests that the groups did not differ in the overall magnitude of displacement of water molecules in the CST.

We did not find any significant change in DTI indices in any other brain regions. This suggests that trauma-induced changes are specific to the sensorimotor system as shown previously. ${ }^{6}$ However, due to the small sample size, weak effects may not have been detected in regions other than the CST. ${ }^{6}$

Most of the significant alterations in DTI indices were found in the right CST innervating the left hand and leg. This asymmetry in DTI findings may be explained by the increased use of the less affected right arm and hand post injury (table 1), which is innervated largely by the left CST. $^{7}$ Thus compensatory (over-) use of a less affected body part ${ }^{42}{ }^{43}$ might have led to increased neurotrophic support-reflected in a higher degree of axonal integrity in the left CST.

\section{Reduced cross-sectional spinal cord area predicts increased RD in the brain}

Moving beyond the group differences, our aim was to determine the relationship between micro, and macrostructural effects of a traumatic SCI. We have previously reported a reduction in SCA of more than $30 \%$ in the same patient cohort. ${ }^{4}$ Here we demonstrate a linear relationship between the observed macroscopic cervical tissue losses (ie, reduced SCA) and increased RD within the right cerebral peduncle following traumatic SCI. Changes in $\mathrm{RD}$ are thought to reflect the integrity of myelin ${ }^{11}$ and to be predictors of clinical recovery. ${ }^{41}$ As a consequence of trauma the spinal cord several segments rostral to the site of initial trauma turns atrophic due to retrograde and anterograde degenerative processes. $^{2} 4$ Therefore, the observed correlation between SCA and increased RD within the CST suggests that trauma-induced spinal degenerative processes progress towards the cerebrum.

In agreement with a previous report, ${ }^{3}$ we did not find a significant correlation between disease duration and altered DTI metrics in chronic SCI. Given that the mean time since injury ranged between 7 and 13 years, the greatest changes might have occurred in the acute and sub acute phase of injury. Thus, the changes we observe might be long standing. Future longitudinal studies will clarify the temporal and spatial patterns of long distance degenerative changes following SCI.

\section{Reduced FA predicts cortical reorganisation}

In order for trauma-induced plasticity to take place, microstructural rearrangements of grey and white matter are required.

Table 2 Correlation between lower cord area and altered diffusion-tensor imaging metrics in spinal cord injury subjects

\begin{tabular}{|c|c|c|c|c|c|c|c|}
\hline & $\begin{array}{l}\text { FA right } \\
\text { pyramids }\end{array}$ & $\begin{array}{l}\text { FA left } \\
\text { pyramids }\end{array}$ & $\begin{array}{l}\text { FA right } \\
\text { int. capsule }\end{array}$ & $\begin{array}{l}\text { FA left } \\
\text { M1 hand }\end{array}$ & $\begin{array}{l}\text { AD right } \\
\text { cer. peduncle }\end{array}$ & $\begin{array}{l}\text { RD right } \\
\text { pyramid }\end{array}$ & $\begin{array}{l}\text { RD right } \\
\text { cer. peduncle }\end{array}$ \\
\hline \multirow[t]{2}{*}{ Cord area } & $R=0.484$ & $R=0.462$ & $\mathrm{R}=0.118$ & $R=0.375$ & $R=0.197$ & $\mathrm{R}=0.355$ & $\mathrm{R}=0.718$ \\
\hline & $p=0.290$ & $p=0.271$ & $p=0.906$ & $p=0.369$ & $p=0.640$ & $p=0.406$ & $p=0.029$ \\
\hline \multirow[t]{2}{*}{ Disease duration } & $R=0.458$ & $R=0.604$ & $R=0.084$ & $R=0.049$ & $\mathrm{R}=0.391$ & $\mathrm{R}=0.186$ & $\mathrm{R}=0.263$ \\
\hline & $p=0.215$ & $p=0.085$ & $p=0.829$ & $p=0.901$ & $p=0.298$ & $p=0.632$ & $p=0.494$ \\
\hline
\end{tabular}

AD, Axial diffusivity; cer, cerebral; FA, Fractional anisotropy; int, internal; RD, Radial diffusivity; R, Pearson's correlation. 
Figure 5 (A) Statistical parametric maps (thresholded at $p<0.01$, uncorrected for display purposes) showing regions of increased taskrelated brain activation during rightsided handgrip in contralateral left M1; (B) Statistical parametric maps (thresholded at $p<0.01$, uncorrected for display purposes) showing the left M1 leg region in which changes in taskrelated blood oxygenation leveldependent activation during handgrip are more sensitive to changes in fractional anisotropy (FA) of the left pyramid following trauma compared with normal variability. The colour bar represents the $t$-value. The scatter plots depict the regression of parameter estimates testing for activations against the FA values in the left pyramids.
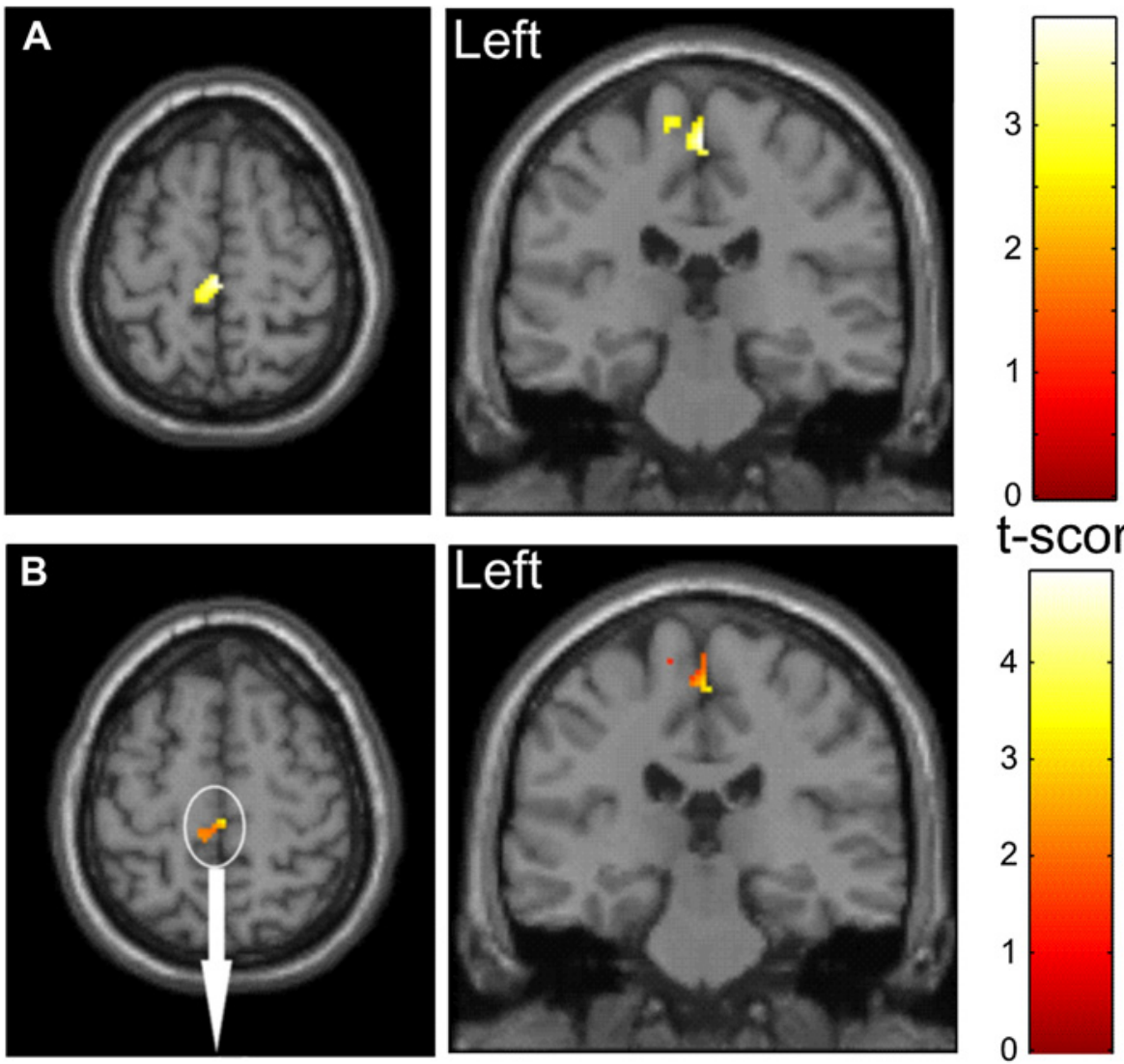

t-score
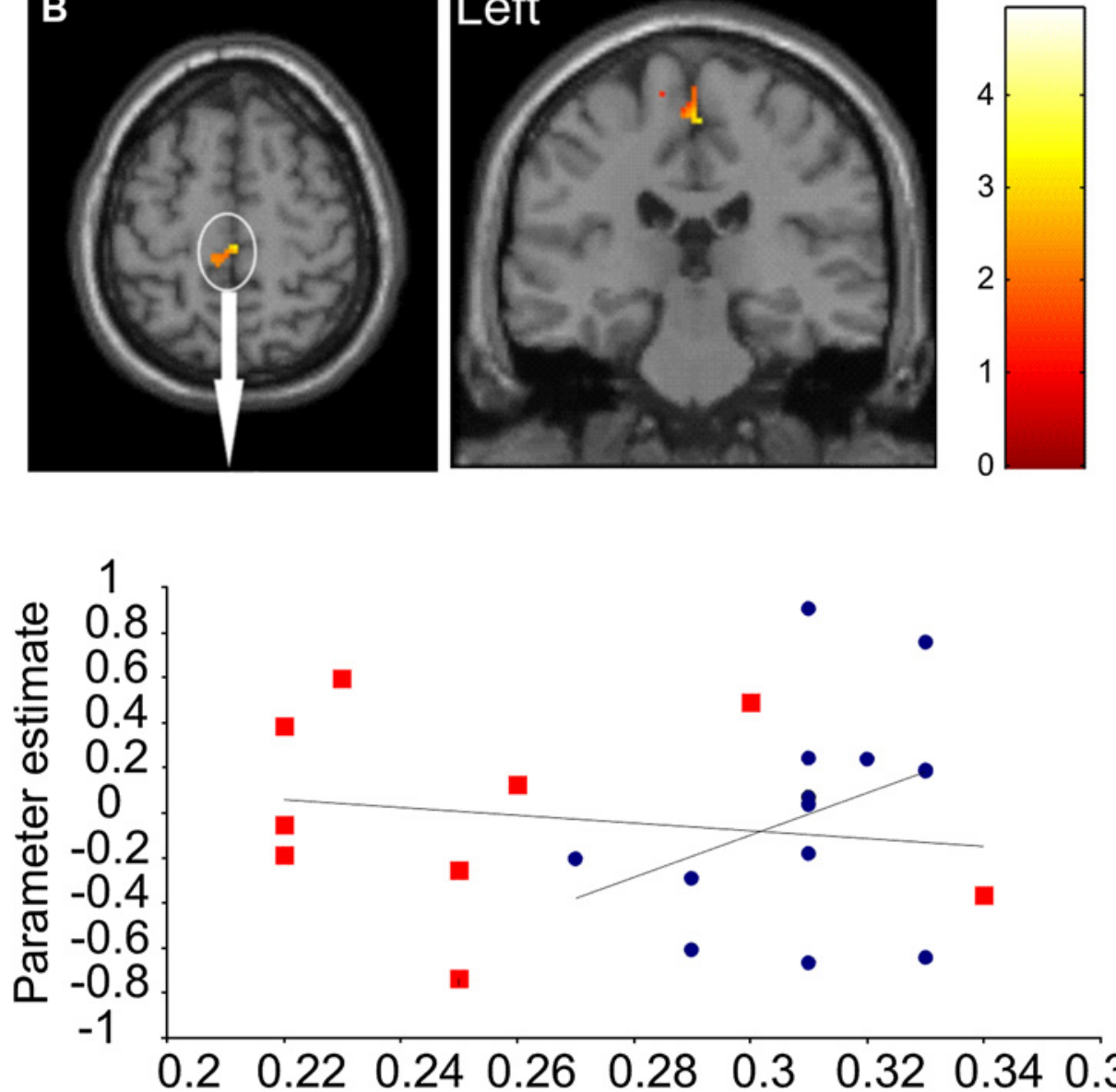

FA in the left pyramid
More recently, another potential mechanism-contributing to cortical reorganisation following SCI-has been proposed. This mechanism involves rewiring of injured CST neurons at a cervical level ${ }^{44}$ and transient down-regulation of the Nogoreceptor-1 signalling cascade ${ }^{45}$ - the key receptor component of the Nogo nerve growth inhibitory signalling system ${ }^{46}$-in response to increased altered cortical neuronal activity. ${ }^{45}$ Besides rewiring and down-regulation of inhibitory signalling cascades, changes in WM microstructure within the pyramids may play an active role in trauma-related plasticity. In the primary sensory cortex, cortical reorganisation of brushing-evoked brain activation of the little finger shifts towards the leg area that is associated with anatomical changes within the same area in thoracic injured patients. ${ }^{13}$

Previously using functional MRI and voxel-based morphometry, we demonstrated, in the same SCI patient group, relative increases in task-related activation during right handgrip and structural associations between measures of manual dexterity and GM volume in the 'paralysed' leg region of left M1. ${ }^{4}$ Here, we extend these findings by showing an interaction between task-related activation during right handgrip in the 'paralysed' leg region of left $\mathrm{M} 1$ and reduced FA in the left pyramid containing the CST. In other words, there is dissociation between SCI subjects and controls; where lower pyramidal FA is 
associated with increased hand grip-related activations in the M1 leg area. Given that the cortex in SCI patients is rendered hyperactive after a $\mathrm{SCI}^{4}$; one possible mechanism underlying this relationship could be subcortical rewiring. ${ }^{44}$ As we are not able to distinguish between leg and hand CST fibres, as they run in close proximity, ${ }^{7}$ we can only speculate that this interaction was driven by rewired CST leg fibres in SCI patients. Crucially, we were able to show that these pathological changes in the CST are above and beyond what one would expect from normal variability in central and spinal anatomy.

However, there are potential confounds that might have induced increased hand grip-related activation in the leg area, which may be unrelated to subcortical structural plasticity. For example, increased task related activation may stem from automatic time-locked inter-limb coupling ${ }^{47}$ or non-specific overflow of the leg area during hand movement, which is normally prevented in normal individuals. ${ }^{15}$ During scanning, we instructed participants to exert $30 \%$ of their maximum voluntary contraction for the handgrip task, we did not observe any co-contractions and we controlled for performance. We are therefore confident that the possible confounds played a minor role in increased activation in the leg area of M1. Thus, we infer that trauma-induced reduction in CST integrity is associated with altered task-related cortical activation during handgrip in the leg representing area of $\mathrm{M} 1$.

\section{Methodological considerations}

Age-related decline in brain WM anisotropy presents a possible confound in this study. ${ }^{48}$ SCI subjects were on an average seven years older than controls but this age difference was not significant at the group level. To account for any influence of the latter on our data, we included age as a regressor of no interest in the regression analysis. This means that the relationships reported above cannot be explained in terms of (linear) agerelated effects.

Problems with implementations of voxel-based analyses of DTI data have been reported in the literature ${ }^{29} 49$ These involve image misregistration, tissue specificity and smoothing. The methods used in this study were selected carefully to finesse these problems. Susceptibility induced geometric distortions were minimised using the data acquisition and processing method of Anderson et al. ${ }^{24}$ allowing for a valid linear coregistration between DTI data and T1w anatomical data. As a consequence, it was possible to accurately identify subjectspecific WM in the DTI maps using the segmented T1w images. ${ }^{50}$ Furthermore, subject DTI data could be registered to a standard space using the T1w images with DARTEL (Diffeomorphic Anatomical Registration using Exponentiated Lie algebra), ${ }^{28}$ which has been demonstrated to provide nearoptimal anatomical precision. ${ }^{51}$ In particular, this methodology overcomes some of the problems with registration of DTI data from different subjects. It also provides confidence in the use of objectively defined regions of interest from an anatomical atlas, even for patients whose neuroanatomy differs from healthy subjects. Finally, we used a method to compensate for the tissuespecific smoothing applied to the spatially normalised data. ${ }^{29}$

\section{CONCLUSION}

In conclusion, we show that axonal and myelin integrity is reduced in spatially specific areas following traumatic SCI. Crucially, we demonstrate that CST integrity is directly correlated with lower SCA and cortical reorganisation. Future longitudinal studies in larger cohorts of SCI subjects are necessary to investigate whether structural and functional imaging markers can be used to monitor disease progression and the effect of treatments targeting the repair of the injured spinal cord.

Acknowledgements The authors would like to thank all participants who generously helped with this study: Dr Angela Gall, Judith Susser and Dr Vernie Balasubramaniam for clinical assessment, the radiographers of the Wellcome Trust Centre for Neuroimaging for technical support and Professor Ray Dolan for critical review of an earlier version of the manuscript.

Contributors PF and $\mathrm{CH}$ designed the study, acquired the data, performed statistical analysis and wrote the paper. CW- $\mathrm{K}, \mathrm{ZN}$ designed the imaging protocol and revised the paper. KF, AJT and NW designed the study, performed statistical analysis and revised the paper.

Funding This work was supported by the Swiss National Science Foundation (Grant No: PBFR33-120920), Schweizerische Stiftung für medizinische und biologische Stipendien (Grant No: PASMP3-124194), Swiss Paraplegic Research (Nottwil) and the Wellcome Trust. This work was undertaken at UCLH/UCL which received a proportion of funding from the Department of Health's NIHR Biomedical Research Centres funding scheme.

\section{Competing interests None.}

Patient consent The content of this paper does not include any personal information referring to individuals. Therefore we guarantee full anonymity. All participants gave informed, written consent before the study, which was approved by the Joint Ethics Committee of the UCL-Institute of Neurology and the National Hospital for Neurology and Neurosurgery (ref: 08/0243).

Ethics approval Ethics approval was granted by Joint Ethics Committee of the UCL-Institute of Neurology and the National Hospital for Neurology and Neurosurgery (ref: 08/0243)

Provenance and peer review Not commissioned; externally peer reviewed.

\section{REFERENCES}

1. Dietz V. Behavior of spinal neurons deprived of supraspinal input. Nat Rev Neurol 2010:6:167-74.

2. Lundell H, Barthelemy D, Skimminge A, et al. Independent spinal cord atrophy measures correlate to motor and sensory deficits in individuals with spinal cord injury. Spinal Cord 2011:49:70-5.

3. Cohen-Adad J, El Mendili MM, Lehericy S, et al. Demyelination and degeneration in the injured human spinal cord detected with diffusion and magnetization transfer MRI. Neuroimage 2011:55:1024-33.

4. Freund P, Weiskopf N, Ward NS, et al. Disability, atrophy and cortical reorganization following spinal cord injury. Brain 2011;134:1610-22.

5. Jurkiewicz MT, Crawley AP, Verrier MC, et al. Somatosensory cortical atrophy after spinal cord injury: a voxel-based morphometry study. Neurology 2006:66:762-4.

6. Wrigley PJ, Gustin SM, Macey PM, et al. Anatomical changes in human motor cortex and motor pathways following complete thoracic spinal cord injury. Cereb Cortex 2009:19:224-32.

7. Lemon RN. Descending pathways in motor control. Annu Rev Neurosci 2008;31:195-218.

8. Basser PJ, Pierpaoli C. Microstructural and physiological features of tissues elucidated by quantitative-diffusion-tensor MRI. J Magn Reson B 1996;111:209-19.

9. Gouw AA, Seewann A, Vrenken $\mathrm{H}$, et al. Heterogeneity of white matter hyperintensities in Alzheimer's disease: post-mortem quantitative MRI and neuropathology. Brain 2008;131:3286-98.

10. Schmierer K, Wheeler-Kingshott CA, Boulby PA, et al. Diffusion tensor imaging of post mortem multiple sclerosis brain. Neuroimage 2007;35:467-77.

11. Zhang J, Jones M, DeBoy CA, et al. Diffusion tensor magnetic resonance imaging of Wallerian degeneration in rat spinal cord after dorsal root axotomy. J Neurosci 2009;29:3160-71.

12. Kim JH, Loy DN, Wang Q, et al. Diffusion tensor imaging at 3 hours after traumatic spinal cord injury predicts long-term locomotor recovery. J Neurotrauma 2010;27:587-98

13. Henderson LA, Gustin SM, Macey PM, et al. Functional reorganization of the brain in humans following spinal cord injury: evidence for underlying changes in cortical anatomy. J Neurosci 2011;31:2630-7.

14. Kokotilo KJ, Eng JJ Curt A. Reorganization and preservation of motor control of the brain in spinal cord injury: a systematic review. J Neurotrauma 2009;26:2113-26.

15. Freund $\mathbf{P}$, Rothwell J, Craggs $\mathrm{M}$, et al. Corticomotor representation to a human forearm muscle changes following cervical spinal cord injury. Eur J Neurosci 2011;34:1839-46.

16. Freund PA, Dalton C, Wheeler-Kingshott CA, et al. Method for simultaneous voxelbased morphometry of the brain and cervical spinal cord area measurements using 3D-MDEFT. J Magn Reson Imaging 2010;32:1242-7.

17. Deichmann R, Schwarzbauer C, Turner R. Optimisation of the 3D MDEFT sequence for anatomical brain imaging: technical implications at 1.5 and 3 T. Neuroimage 2004;21:757-67. 
18. Losseff NA, Webb SL, O'Riordan JI, et al. Spinal cord atrophy and disability in multiple sclerosis. A new reproducible and sensitive MRI method with potential to monitor disease progression. Brain 1996;119:701-8.

19. Weiskopf N, Hutton C, Josephs 0 , et al. Optimal EPI parameters for reduction of susceptibility-induced BOLD sensitivity losses: a whole-brain analysis at $3 \mathrm{~T}$ and 1.5 T. Neuroimage 2006:33:493-504.

20. Ward NS, Frackowiak RS. Age-related changes in the neural correlates of motor performance. Brain 2003;126:873-88.

21. Andersson JL, Hutton C, Ashburner J, et al. Modeling geometric deformations in EPI time series. Neuroimage 2001;13:903-19.

22. Friston KJ, Holmes AP, Worsley KJ, et al. Statistical parametric maps in functional imaging: a general linear approach. Hum Brain Mapp 1995;2:189-210.

23. Reese TG, Heid 0, Weisskoff RM, et al. Reduction of eddy-current-induced distortion in diffusion MRI using a twice-refocused spin echo. Magn Reson Med 2003:49:177-82.

24. Andersson JL, Skare S, Ashburner J. How to correct susceptibility distortions in spin-echo echo-planar images: application to diffusion tensor imaging. Neuroimage 2003;20:870-88.

25. Jansons KM, Alexander DC. Persistent angular structure: new insights from diffusion MRI data. Dummy version. Inf Process Med Imaging 2003:18:672-83.

26. Chang LC, Jones DK, Pierpaoli C. RESTORE: robust estimation of tensors by outlier rejection. Magn Reson Med 2005;53:1088-95.

27. Cook PA, Bai Y, Nedjati-Gilani S, et al. Camino: open-cource diffusion-MR reconstruction and processing. Proc Int Soc Mag Reson Med 2006;14:2759. [abstract]Cook PA, Bai Y, Nedjati-Gilani S, et al.

28. Ashburner J. A fast diffeomorphic image registration algorithm. Neuroimage 2007;38:95-113

29. Lee JE, Chung MK, Lazar M, et al. A study of diffusion tensor imaging by tissue-specific, smoothing-compensated voxel-based analysis. Neuroimage 2009;44:870-83.

30. Wakana S, Caprihan A, Panzenboeck MM, et al. Reproducibility of quantitative tractography methods applied to cerebral white matter. Neuroimage 2007;36:630-44.

31. Mori S, Oishi $\mathrm{K}$, Jiang $\mathrm{H}$, et al. Stereotaxic white matter atlas based on diffusion tensor imaging in an ICBM template. Neuroimage 2008;40:570-82.

32. Kapreli E, Athanasopoulos S, Papathanasiou M, et al. Lateralization of brain activity during lower limb joints movement. An fMRI study. Neuroimage 2006;32:1709-21.

33. Barkhof F, Calabresi PA, Miller DH, et al. Imaging outcomes for neuroprotection and repair in multiple sclerosis trials. Nat Rev Neurol 2009:5:256-66.

34. Kozlowski P, Raj D, Liu J, et al. Characterizing white matter damage in rat spinal cord with quantitative MRI and histology. J Neurotrauma 2008;25:653-76.
35. DeBoy CA, Zhang J, Dike $S$, et al. High resolution diffusion tensor imaging of axonal damage in focal inflammatory and demyelinating lesions in rat spinal cord. Brain 2007:130:2199-210.

36. Wheeler-Kingshott CA, Cercignani M. About "axial" and "radial" diffusivities. Magn Reson Med 2009;61:1255-60.

37. Beaulieu C, Does MD, Snyder RE, et al. Changes in water diffusion due to Wallerian degeneration in peripheral nerve. Magn Reson Med 1996;36:627-31.

38. Blight AR, DeCrescito V. Morphometric analysis of experimental spinal cord injury in the cat: the relation of injury intensity to survival of myelinated axons. Neuroscience 1986:19:321-41.

39. Budde MD, Xie M, Cross AH, et al. Axial diffusivity is the primary correlate of axonal injury in the experimental autoimmune encephalomyelitis spinal cord: a quantitative pixelwise analysis. J Neurosci 2009;29:2805-13

40. Song SK, Yoshino J, Le TQ, et al. Demyelination increases radial diffusivity in corpus callosum of mouse brain. Neuroimage 2005:26:132-40.

41. Freund $\mathbf{P}$, Wheeler-Kingshott $\mathbf{C}$, Jackson $\mathrm{J}$, et al. Recovery after spinal cord relapse in multiple sclerosis is predicted by radial diffusivity. Mult Scler 2010:16:1193-202.

42. Elbert $\mathrm{T}$, Flor $\mathrm{H}$, Birbaumer $\mathrm{N}$, et al. Extensive reorganization of the somatosensory cortex in adult humans after nervous system injury. Neuroreport 1994:5:2593-7.

43. Nudo RJ, Wise BM, SiFuentes F, et al. Neural substrates for the effects of rehabilitative training on motor recovery after ischemic infarct. Science 1996:272:1791-4.

44. Ghosh A, Haiss F, Sydekum E, et al. Rewiring of hindlimb corticospinal neurons afte spinal cord injury. Nat Neurosci 2010;13:97-104.

45. Endo T, Spenger C, Tominaga $T$, et al. Cortical sensory map rearrangement after spinal cord injury: $\mathrm{fMRI}$ responses linked to nogo signalling. Brain 2007:130:2951-61

46. Gonzenbach RR, Schwab ME. Disinhibition of neurite growth to repair the injured adult CNS: focusing on nogo. Cell Mol Life Sci 2008;65:161-76.

47. Calancie B, Lutton S, Broton JG. Central nervous system plasticity after spinal cord injury in man: interlimb reflexes and the influence of cutaneous stimulation. Electroencephalogr Clin Neurophysiol 1996;101:304-15.

48. Pfefferbaum A, Sullivan EV, Hedehus M, et al. Age-related decline in brain white matter anisotropy measured with spatially corrected echo-planar diffusion tensor imaging. Magn Reson Med 2000:44:259-68.

49. Smith SM, Jenkinson $\mathrm{M}$, Johansen-Berg $\mathrm{H}$, et al. Tract-based spatial statistics: voxelwise analysis of multi-subject diffusion data. Neuroimage 2006;31:1487-505

50. Ashburner J, Friston KJ. Unified segmentation Neuroimage 2005;26:839-51.

51. Klein A, Andersson J, Ardekani BA, et al. Evaluation of 14 nonlinear deformation algorithms applied to human brain MRI registration. Neuroimage 2009;46:786-802. 